\title{
SNPs at miR-155 binding sites of TYRP1 explain discrepancy between mRNA and protein and refine TYRP1 prognostic value in melanoma
}

\author{
P El Hajj ${ }^{1}$, D Gilot ${ }^{2}$, M Migault ${ }^{2}$, A Theunis ${ }^{3}, L$ C van Kempen ${ }^{4}$, F Salés ${ }^{1}$, H Fayyad-Kazan ${ }^{5}$, B Badran ${ }^{6}$,

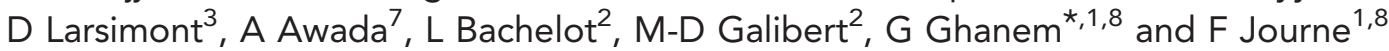

${ }^{1}$ Laboratory of Oncology and Experimental Surgery, Institut Jules Bordet, Université Libre de Bruxelles, 1 Rue Heger-Bordet, 1000 Brussels, Belgium; ${ }^{2}$ CNRS UMR 6290, Université de Rennes 1, 2 Avenue du Pr. Léon Bernard, 35000 Rennes, France; ${ }^{3}$ Department of Pathology, Institut Jules Bordet, Université Libre de Bruxelles, 1 Rue Heger-Bordet, 1000 Brussels, Belgium; ${ }^{4}$ Department of Pathology, McGill University and Lady Davis Institute for Medical Research, Jewish General Hospital, 3755 Chemin de la Côte-Sainte-Catherine, H3T 1E2 Montreal, QC, Canada; ${ }^{5}$ Laboratory of Experimental Hematology, Institut Jules Bordet, Université Libre de Bruxelles, 1 Rue Heger-Bordet, 1000 Brussels, Belgium; ' Department of Biochemistry, Lebanese University, Rafic Campus, 1003 Hadath-Beirut, Lebanon and ${ }^{7}$ Clinic of Medical Oncology, Institut Jules Bordet, Université Libre de Bruxelles, 1 Rue Heger-Bordet, 1000 Brussels, Belgium

Background: We previously demonstrated an inverse correlation between tyrosinase-related protein 1 (TYRP1) mRNA expression in melanoma metastases and patient survival. However, TYRP1 protein was not detected in half of tissues expressing mRNA and did not correlate with survival. Based on a study reporting that $3^{\prime}$ untranslated region (UTR) of TYRP1 mRNA contains two miR-155$5 p$ (named miR-155) binding sites exhibiting single-nucleotide polymorphisms (SNPs) that promote (matched miRNA-mRNA interaction) mRNA decay or not (mismatched), we aimed to investigate the role of miR-155 in the regulation of TYRP1 mRNA expression and protein translation accounting for these SNPs.

Methods: The effect of miR-155 on TYRP1 mRNA/protein expression was evaluated in two melanoma cell lines harbouring matched or mismatched miR-155-TYRP1 mRNA interaction after transfection with pre-miR-155. In parallel, 192 skin and lymph node melanoma metastases were examined for TYRP1 mRNA/protein, miR-155 and SNPs and correlated with patient survival. TYRP1 mRNA, SNPs at its 3'UTR and miR-155 were analysed by RT-qPCR, whereas TYRP1 protein was evaluated by western blot in cell lines and by immunohistochemistry in metastatic tissues.

Results: The miR-155 induced a dose-dependent TYRP1 mRNA decay and hampered its translation into protein in the line with the 'match' genotype. In melanoma metastases, TYRP1 mRNA inversely correlated with miR-155 expression but not with TYRP1 protein in the 'match' group, whereas it positively correlated with protein but not with miR-155 in the 'mismatch' group. Consequently, in the latter group, TYRP1 protein inversely correlated with survival.

Conclusion: Polymorphisms in 3'UTR of TYRP1 mRNA can affect TYRP1 mRNA regulation by miR-155 and its subsequent translation into protein. These SNPs can render TYRP1 mRNA and protein expression nonsusceptible to miR-155 activity and disclose a prognostic value for TYRP1 protein in a subgroup of melanoma patients. These data support the interest in the prognostic value of melanogenic markers and propose TYRP1 to refine prognosis in patients with advanced disease.

*Correspondence: Professor G Ghanem; E-mail: gghanem@ulb.ac.be

${ }^{8}$ These authors are joint senior authors.

Received 22 December 2014; revised 19 March 2015; accepted 29 April 2015; published online 11 June 2015

(c) 2015 Cancer Research UK. All rights reserved 0007-0920/15 
Cutaneous melanoma is the most lethal form of skin cancer with a variable clinical outcome even in patients with in situ primary lesions or thin melanomas. The variability in survival of patients with stage III (39-70\% for 5-year survival) and stage IV melanoma (33-62\% for 1-year survival) (Balch et al, 2009) points to an insufficient understanding of the heterogeneity of the disease and warns of difficulties to select patients who could benefit from treatments. It is thus of importance to identify genes/proteins that may be used as new molecular markers for prognosis as well as new potential targets for therapy.

Many studies demonstrated a significant association of melanoma markers with disease-free survival (DFS) (Meyer et al, 2012) or overall survival (OS) (Kashani-Sabet et al, 2009). A systematic review of published immunohistochemistry (IHC)based biomarkers was conducted to identify proteins as prognostic biomarkers of patient outcomes (Gould Rothberg et al, 2009). However, the majority of identified markers have not been shown to improve the current AJCC staging (Balch et al, 2009), preventing their translation into routine clinical assessment.

We recently showed a significant association between tyrosinase-related protein 1 (TYRP1) mRNA expression in skin and lymph node metastases of stage III and IV melanoma patients and survival (Journe et al, 2011; El Hajj et al, 2013). Indeed, high TYRP1 mRNA level significantly correlated with both reduced DFS and OS. These data suggested that the evaluation of TYRP1 may be used to refine the initial prognosis defined at the time of primary and may dictate a different management of the advanced disease.

The TYRP1 is an enzyme involved in pigmentation and is abundantly expressed in melanocytes and pigmented melanoma cells. Its enzymatic function, however, has not been clearly elucidated (no good substrate) in human melanocytes where it might have weak oxidase or catalase activities. Its expression has been associated with melanoma progression and aggressiveness through an increase of tyrosinase stability and cell protection against toxic melanin intermediates (reviewed in Ghanem and Fabrice, 2011).

In our former study (Journe et al, 2011), we also checked for TYRP1 protein expression by IHC in a panel of paraffin-embedded biopsies from skin metastases and found a lack of association with patient survival. We also reported discrepancies with the TYRP1 mRNA levels measured by RT-qPCR in corresponding frozen tissues in $\sim 50 \%$ of the cases where mRNA is expressed but not the protein, suggesting post-transcriptional regulation(s).

MicroRNAs (miRNAs) are endogenous noncoding small RNAs that bind to the $3^{\prime}$ untranslated region (UTR) of target mRNAs and post-transcriptionally modulate the products of gene expression by promoting transcript degradation and/or by negatively regulating translational efficiency of their target mRNAs. It is predicted that $\sim 60 \%$ of all human protein-coding genes are regulated by miRNAs (Sayed and Abdellatif, 2011). Therefore, miRNAs are of great importance in diverse physiological and developmental processes in humans (Osman, 2012).

Single-nucleotide polymorphisms (SNPs) are common genetic variants in the human genome (Reich et al, 2003) and when they occur in the $3^{\prime}$ UTR of mRNAs, they can affect the binding capacity of microRNAs. In this context, Li et al (2012) suggested that miR155-5p (named miR-155 within the text) acts as a 'rheostat' to optimise TYRP1 expression for local adaptation to differential UV radiation along the latitudes. Moreover, the authors showed that the $3^{\prime}$ UTR of TYRP1 mRNA contains three putative miR-155 binding sites, among which two are polymorphic (SNPs rs683/ rs910). The derived alleles (CC/AA combination) form intact microRNA sites with matched miRNA-target interaction and high binding capacity in the African and Asian population, leading to TYRP1 mRNA decay and translation repression by miR-155. In contrast, two-thirds of Utah residents with European ancestry carry the ancestral alleles (AA/CC combination) disrupting the
miRNA-target interaction and thus limiting miR-155 regulation on TYRP1. They also confirmed the direct interaction with miR- 155 by luciferase assays, especially for rs 683 that mediated the strongest suppression effect.

In the light of our previous finding reporting discrepancy between TYRP1 protein and mRNA expression in skin metastases and the potential regulation of TYRP1 expression by miRNAs, we aim to investigate the role of the miR-155 in the regulation of TYRP1 mRNA expression and translation: first, in two melanoma cell lines transfected with pre-miR-155 and, second, in 192 melanoma skin and lymph node metastases addressing the prognostic value of TYRP1 in a SNP-dependent manner.

\section{MATERIALS AND METHODS}

Patients and tissue collection. Skin $(N=82)$ and lymph node $(N=110)$ metastases were collected from patients with stage III and IV melanoma undergoing surgery at Institut Jules Bordet (Brussels, Belgium) from 1998 to 2009. Samples were collected randomly with no inclusion or exclusion criteria. All lymph node metastases were palpable lymph node macrometastases confirmed by sonography and pathology examination. Immediately after surgery, half of each biopsy was fixed in formalin, embedded in paraffin, sectioned according to routine clinical procedures and was used for IHC or snap-frozen in liquid nitrogen and stored at $-80^{\circ} \mathrm{C}$ until RNA extraction. This study was approved by the ethic committee of Institut Jules Bordet (CE1959) and performed in accordance with the REMARK guidelines (Alonzo, 2005; McShane et al, 2005). The clinical characteristics of the patients are outlined in Table 1.

RNA extraction and RT-qPCR. Total RNA was extracted, and TYRP1, S100B and MITF mRNA expressions were quantified by RT-qPCR as previously described (El Hajj et al, 2013; Primot et al, 2010) and according to MIQE guidelines (Bustin et al, 2009). Relative quantification in frozen tissues was determined by normalising the cycle threshold (CT) of TYRP1 with the CT of S100B (loading control) using the $2^{-\Delta C T}$ method. Relative expression in cell lines was calculated by normalising the CT of TYRP1 with the CT of RPS18 (loading control) using the $2^{-\Delta C T}$ method. Indeed, evaluating the expression of several mRNAs (CASC3, RPS18, GAPDH, ACTB) as loading control, we found that RPS18 had the least variation of CT in 20 cell lines. Primer sequences for RPS18 mRNA detection were designed using Primer 3 (http://frodo.wi.mit.edu) (forward: 5'-GCGGCGGAAA ATAGCCTTTG-3', reverse: 5'-GATCACACGTTCCACCTCAT C-3', amplicon size: 139, efficiency: 99\%) (Life Technologies, Gent, Belgium).

MicroRNA RT-qPCR. Total RNA concentrations were determined by the NanoDrop 1000 spectrophotometer (Thermo Scientific, Wilmington, DE, USA). The RNA was reverse transcribed using the TaqMan MicroRNA Reverse Transcription Kit and miR-155 was assessed by RT-qPCR using the TaqMan MicroRNA Assay Kit (PN 4427975, catalogue numbers 002623 for miR-155-5p and 001094 for RNU44; Applied Biosystems, Ghent, Belgium). This stem-loop RT-qPCR method detects specifically mature, but not precursor, miRNA. Briefly, 10 ng of template RNA was reverse transcribed using miRNA-specific stem-loop primers for $30 \mathrm{~min}$ at $16^{\circ} \mathrm{C}, 30 \mathrm{~min}$ at $42^{\circ} \mathrm{C}$ and $5 \mathrm{~min}$ at $85^{\circ} \mathrm{C}$. The PCR reactions were run on ABI Prism $7900 \mathrm{HT}$ Sequence Detection System (Applied Biosystems) at $95^{\circ} \mathrm{C}$ for $10 \mathrm{~min}$ followed by 40 cycles at $95^{\circ} \mathrm{C}$ for $15 \mathrm{~s}$ and $60{ }^{\circ} \mathrm{C}$ for $1 \mathrm{~min}$. All PCR reactions were performed in duplicate. Relative evaluation was calculated by normalising the CT of miR-155 with the CT of RNU44, a small nucleolar RNA, using the $2^{-\Delta \mathrm{CT}}$ method. 
Table 1. Patient and sample characteristics

\begin{tabular}{|c|c|c|c|}
\hline Parameters & $N$ & Median & Range \\
\hline \multicolumn{4}{|l|}{ Patients } \\
\hline Site of metastasis (lymph node/skin) & $110 / 82$ & & \\
\hline Gender (female/male) & $111 / 81$ & & \\
\hline Age $\left(\right.$ years) ${ }^{a}$ & 186 & 55 & $20-87$ \\
\hline Stage $(I I I / I V)^{b}$ & $136 / 55$ & & \\
\hline Breslow $(\mathrm{mm})^{c}$ & 169 & 2.6 & $0.3-45.0$ \\
\hline Ulceration (no/yes) ${ }^{d}$ & $42 / 58$ & & \\
\hline OS (years) & 185 & 4.7 & $0.8-31.1$ \\
\hline \multicolumn{4}{|l|}{ Samples } \\
\hline MITF mRNA & 191 & 0.39 & $0-114.2$ \\
\hline \multicolumn{4}{|l|}{ SNPs rs683/rs910 } \\
\hline CC/AA & 48 & & \\
\hline $\mathrm{AA} / \mathrm{CC}$ & 101 & & \\
\hline Hetero & 25 & & \\
\hline $\operatorname{miR}-155^{e}$ & 184 & 0.05 & $0.00009-41.9$ \\
\hline TYRP1 mRNA ${ }^{\mathbf{e}}$ & 192 & 0.008 & $0-194$ \\
\hline TYRP1 proteing & 118 & 0 & $0-8$ \\
\hline \multicolumn{4}{|c|}{ 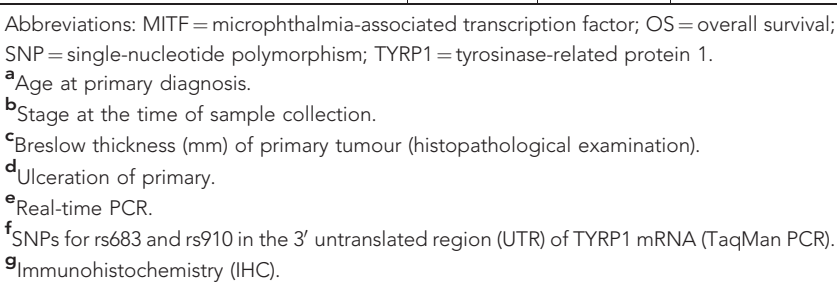 } \\
\hline
\end{tabular}

SNP genotyping. The identification of SNPs for rs683 and rs910 in the $3^{\prime}$ UTR of TYRP 1 mRNA was realised by TaqMan PCR using two different fluorescent reporter probes (Applied Biosystems, Saint Aubin, France). One probe is conjugated with VIC fluorescence dye to monitor transcripts carrying the alleles A for rs683 and rs910. The other probe is conjugated with FAM fluorescence dye to detect the alleles $\mathrm{C}$ for rs683 and rs910, leading to the evaluation of the homozygous CC/AA and AA/CC combinations or the heterozygous one. The RT-qPCR reactions were performed in duplicate in 96-well plate using the mix TaqMan Universal Master Mix II, No AmpErase UNG (Applied Biosystems). Amplification was performed on the 7900HT RT-qPCR System (Applied Biosystems). Sequenced DNA homozygous or heterozygous for the SNP rs683 and rs910 were used as controls for each analysis (3 ng per reaction, NA18486, NA18501, NA18917; Coriell, Camden, NJ, USA).

Immunohistochemistry. Immunohistochemistry for TYRP1 protein was carried out in 118 paraffin-embedded skin and lymph node metastases using the G3E6 antibody raised against the C-terminal TYRP1 (1:100, Abcam, Cambridge, UK) and the ultraView Universal Alkaline Phosphatase Red Detection Kit (Ventana, Tucson, AZ, USA) on the BenchMark XT System (Ventana). The sections were counterstained with haematoxylin (Ventana). Immunostaining was blindly examined by two independent pathologists. A score from 0 to 8 was calculated by adding a score reflecting the proportion of positively stained cells ( non $=0 ; \leq 1 / 100=1 ; 1 / 100$ to $1 / 10=2 ; 1 / 10$ to $1 / 3=3 ; 1 / 3$ to $2 / 3=4$; and $\geq 2 / 3=5$ ) to a score reflecting the staining intensity (none $=0$; weak $=1$; intermediate $=2$; and strong $=3$ ).

Cell culture conditions. Two melanoma cell lines expressing high level of TYRP1 mRNA and low level of endogenous miR-155 were selected for transfection study with regard to genotype: the CC/AA ME1402 line and the AA/CC MM031 one. Cells were routinely cultured at $37^{\circ} \mathrm{C}$ in a humidified $95 \%$ air and $5 \%$ carbon dioxide atmosphere and propagated in flasks containing HAM-F10 medium supplemented with $5 \%$ heat-inactivated fetal calf serum,
5\% heat-inactivated newborn calf serum and with L-glutamine, penicillin and streptomycin at standard concentrations (all from Gibco, Life Technologies). Lines are regularly checked for mycoplasma contamination using MycoAlert Mycoplasma Detection Kit (Lonza, Rockland, ME, USA).

Cell transfection. To achieve an upregulation of miR-155, cell lines were seeded in Petri dishes $\left(1.5 \times 10^{6}\right.$ cells per dish $)$ and cultured for $24 \mathrm{~h}$. Then, cells were transfected for $24 \mathrm{~h}$ with $0.1,0.5$, 1, 2 and 5 nm Pre-miR hsa-miR-155 miRNA Precursor (Pre-miR155, AM17102) or Pre-miR miRNA Precursor Negative Control 1 (Scramble, AM17100; Ambion, Austin, TX, USA), diluted with Lipofectamine RNAiMax Transfection Reagent in Opti-MEM Reduced Serum Medium (both from Life Technologies) according to the manufacturer's protocol. Nontransfected cells served as control. The transfection medium was replaced with fresh HAM-F10 medium and cells were cultured for 3 additional days. Finally, cells were harvested for either RNA isolation or protein extraction to assess TYRP1 mRNA and protein expression by RT-qPCR and western blotting, respectively.

Western blot analysis. Cells were lysed using detergent cocktail (M-PER mammalian extraction buffer) supplemented with protease inhibitors (Halt protease inhibitor cocktail) and phosphatase inhibitors (Halt phosphatase inhibitor cocktail; all from Pierce, Rockford, IL, USA). Protein concentrations were determined by the BCA protein assay (Pierce) using bovine serum albumin as a standard. Equal amounts of cell proteins $(30 \mu \mathrm{g})$ were subjected to $10 \%$ SDS-PAGE and electrotransferred onto nitrocellulose membranes using iBlot Dry Blotting System (Life Technologies). Immunodetection was performed using antibodies raised against TYRP1 (AB23, 1:1000, Santa Cruz Biotechnology, Santa Cruz, CA, USA) and $\beta$-actin (C4, $1: 5000$, Millipore, Temecula, CA, USA). Peroxidase-labelled anti-mouse IgG antibody (1:5000; Amersham Pharmacia Biotech, Roosendaal, The Netherlands) was used as secondary reagents to detect corresponding primary antibodies. Bound peroxidase activity was revealed using the SuperSignal West Pico Chemiluminescent Substrate (Pierce). Immunostaining signals were digitalised with a PC-driven LAS-3000 CCD camera (Fujifilm, Tokyo, Japan), using a software specifically designed for image acquisition (Image Reader, Raytest, Straubenhardt, Germany). Image acquisition was completed before the saturation of pixels providing linearity across a dynamic range of four orders of magnitude. Immunoreactive band intensities were quantified using the software AIDA Image Analyser 3.45 (Raytest).

Statistical analysis. Linear regression was calculated to assess the relationship between TYRP1 (mRNA and protein) and miR-155 expression. Statistical significance between two independent groups was examined using Mann-Whitney test. Statistical correlation between two continuous variables was assessed using Spearman's rho test. Overall survival was estimated using the Kaplan-Meier method. Univariate analyses were performed by Cox regression model to estimate hazard ratios (HRs) and $95 \%$ confidence intervals (CIs). $P$-values of $<0.05$ were considered as statistically significant. All statistical analyses were performed using SPSS15.0, Inc. (Chicago, IL, USA). In order to determine a cutoff point and to categorise patients into two OS groups, we used the Cutoff Finder software available on http://molpath. charite.de/cutoff (Budczies et al, 2012) to optimise cutoff calculation as the point with the most significant (log-rank test) split based on survival variable.

\section{RESULTS}

In vitro evaluation of TYRP1 regulation by miR-155 according to SNPs. According to the data from Li et al (2012), we first 
demonstrated that TYRP1 mRNA/protein expression is affected by high miR-155 levels depending on SNPs within the $3^{\prime} \mathrm{UTR}$ of TYRP1 mRNA. We used the melanoma cell line ME1402 with the CC/AA alleles at SNPs rs683/rs910 reported to promote mRNA decay, and the cell line MM031 harbouring the AA/CC alleles outlined to disrupt it because of mismatches between miR-155 and its target mRNA. Both lines were transfected with increasing concentrations of pre-miR-155 and the intracellular miR-155 levels were assessed by RT-qPCR (Figure 1A). Although ME1402 line expresses lower levels of transfected miR-155 than MM031 line, we observed that the CC/AA ('match') genotype of the former line contributed to a much higher miR-155-induced concentrationdependent decay of TYRP1 mRNA (Figure 1B) and an even more pronounced decline of TYRP1 protein (Figure 1C and D) following miR-155 overexpression compared with the AA/CC ('mismatch') genotype in MM031 line. Indeed, in MM031, TYRP1 mRNA and protein expressions decreased weakly and similarly along with the increase in miR-155 intracellular level, although whereas in ME1402, the decrease of TYRP1 mRNA and protein were, respectively, 3- and 5.8-fold higher compared with MM031.

Characteristics of melanoma patients. The clinical and pathological characteristics of 192 melanoma patients whose skin and lymph node metastases have been evaluated in this study are summarised in Table 1. Overall survival ranged from 0.8 to 31.1 years with a median of 4.7 years. Seven patients (3.6\%) were lost to follow-up for OS. In all, 141 patients (73.4\%) deceased from

A

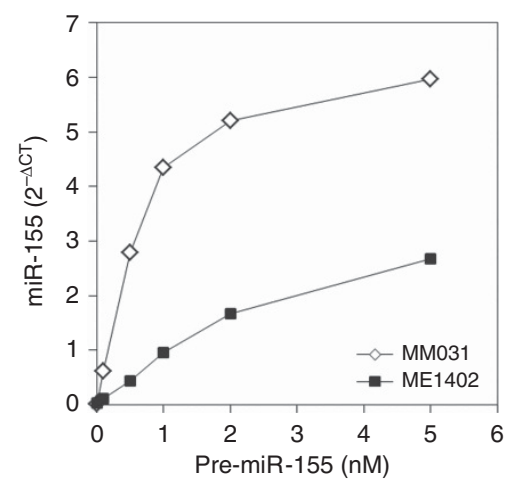

C

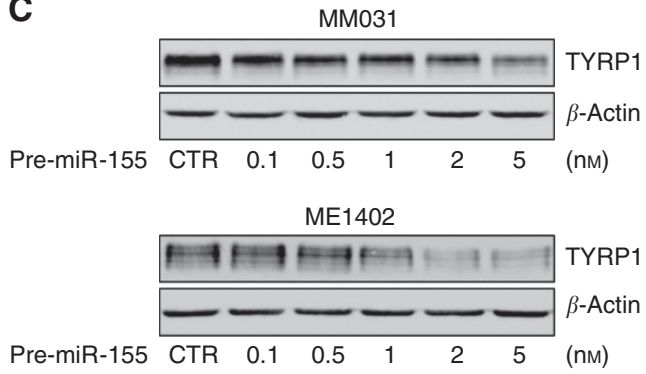

melanoma at the time of the study, and $44(23 \%)$ were still alive. A total of 48 patients $(27.6 \%)$ had the CC/AA alleles at SNPs rs683/rs910, 101 (58\%) had the AA/CC alleles and 25 (14.4\%) were heterozygous. The TYRP1 mRNA, TYRP1 protein and miR-155 were evaluated in 192, 118 and 184 samples, respectively, among which 174, 105 and 169 samples were available for SNP genotyping.

Assessment of TYRP1 protein expression in metastases. The expression of TYRP1 protein was examined by IHC in a panel of 118 paraffin-embedded biopsies from skin $(n=34)$ and lymph node $(n=84)$ metastases and scored from 0 to 8 (Figure 2). Of note, the red staining developed with Fast Red allowed to easily distinguish the immunostaining from black/brown melanin pigments. Examining the whole population, we found that TYRP1 protein was not detected $($ score $=0)$ in 69 samples (Figure 3A), whereas, among this TYRP1 protein-negative tissues, 49 had high levels of TYRP1 mRNA $\left(2^{-\Delta \mathrm{CT}}>0.00015\right.$, selected by using Cutoff Finder software to assess optimal cutoff for patient survival) as measured by RT-qPCR in corresponding frozen tissues. We discarded the hypothesis of an impaired protein detection with the G3E6 antibody (against C-terminus) in 51\% (49 out of 95) of TYRP1 mRNA-positive metastases by assessing two additional antibodies (TRP1 (23) against $\mathrm{N}$-terminus and TA99 against the whole protein; both from Santa Cruz Biotechnology) revealing identical immunostaining profiles (data not shown). Another hypothesis to explain the discrepancy between TYRP1 protein and
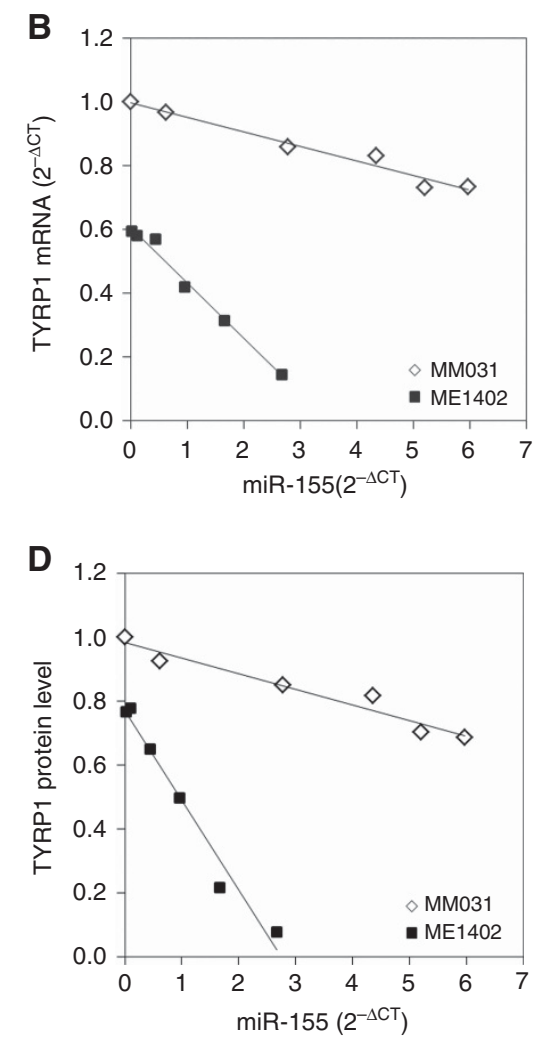

Figure 1. Evaluation of TYRP1 regulation by miR-155 according to SNPs in melanoma cell lines. Measurement of TYRP1 mRNA and protein in ME1402 (CC/AA, 'match' genotype) and MM031 (AA/CC, 'mismatch' genotype) melanoma cell lines transfected with increasing concentrations of either pre-miR scramble (negative control) or pre-miR-155. (A) The miR-155 levels normalised to RNU44 $\left(2^{-\Delta C T}\right.$ ) as function of pre-miR-155 used for transfection (basal level of miR-155 is 0.01 in MM031 and 0.03 in ME1402 line). (B) The TYRP1 mRNA expression normalised to RPS18 (2 ${ }^{-\Delta C T}$ ) as function of miR-155 measured in cells after transfection. (C) Representative western blots (WB) of TYRP1 protein ( $\beta$-actin as loading control) as function of the amount of pre-miR-155 used for transfection. (D) The TYRP1 protein WB bands normalised to $\beta$-actin as function of the amount of miR-155 measured in cells after transfection. All values are the mean of seven independent transfection experiments. No significant difference was found between data from pre-miR scramble transfected cells and nontransfected (control) cells. The TYRP1 mRNA and protein levels are normalized to values from control MM031 cells. Linear regression is used to assess the relationship between mRNA or protein and miR-155 levels. 
mRNA levels could be post-transcriptional regulation(s). Indeed, we found a significant correlation between TYRP1 mRNA and its transcription factor MITF (Table $1 ; \rho=0.53, P<0.001$, Spearman's rho), excluding differential regulation of TYRP1 at transcriptional level.

Association between TYRP1 protein expression and SNP genotype. We learned from $\mathrm{Li}$ et al (2012) and from our in vitro data that the $3^{\prime} \mathrm{UTR}$ of TYRP1 mRNA contains two binding sites for miR-155 that are polymorphic (rs683/rs910), affecting mRNA expression and its translation into protein. We evaluated such SNPs in 174 melanoma metastases and categorised into homozygous AA/CC $(n=101)$, homozygous CC/AA $(n=48)$ groups and heterozygous group $(n=25)$ group (Table 1$)$. First, we observed that TYRP1 protein score is significantly different between the two homozygous genotype groups CC/AA and AA/CC ( $P=0.032$, Mann-Whitney test). Then, by comparing the three groups regarding TYRP1 protein expression, we found a significant difference between the homozygous AA/CC and the heterozygous groups $(P=0.048)$ but none between the latter and the homozygous CC/AA $(P=0.907)$. Therefore, we decided to combine the heterozygous and the homozygous CC/AA ('match') genotypes and found a significant association of this combined group with a low TYRP1 protein expression compared with the homozygous AA/CC ('mismatch') group $(P=0.017$; Figure 3D). No other significant association was found between SNP genotype and either TYRP1 mRNA expression or miR-155 level no matter which group was considered (Figure $3 \mathrm{E}$ and $\mathrm{F}$ ). These data indicate
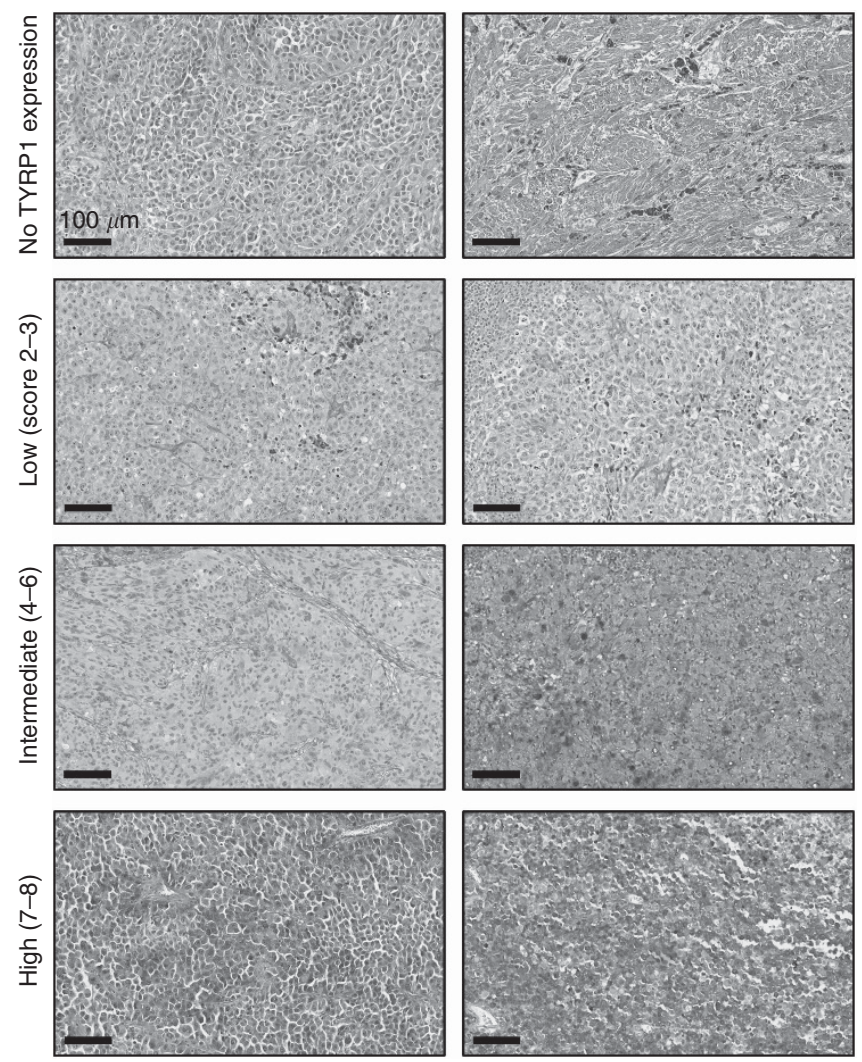

Figure 2. Representative IHC of TYRP1 protein in melanoma metastatic tissues. A total of 118 samples were examined by light microscopy blindly and independently by two pathologists. A score from 0 to 8 was calculated reflecting both the percentage of positively stained cells and the staining intensity. Cytoplasmic red staining was considered as positive. Black/brown granules are melanin pigments. The full colour version of this figure is available at British Journal of Cancer journal online. that the SNPs in the $3^{\prime} \mathrm{UTR}$ of TYRP1 mRNA are involved in the regulation of TYRP1 protein expression.

Differential TYRP1 regulation in melanoma metastases according to SNPs. To better understand TYRP1 regulation in melanoma metastases, we evaluated correlations between miR155, TYRP1 mRNA and TYRP1 protein according to the genotype groups defined above (homo CC/AA plus hetero as 'match' group vs homo AA/CC as 'mismatch' group) using Spearman's rho test. In the 'match' group, we observed a significant inverse correlation between miR-155 and TYRP1 mRNA $(P=0.023)$ but not between TYRP1 mRNA and protein $(P=0.071$; Figure $4 A)$, further
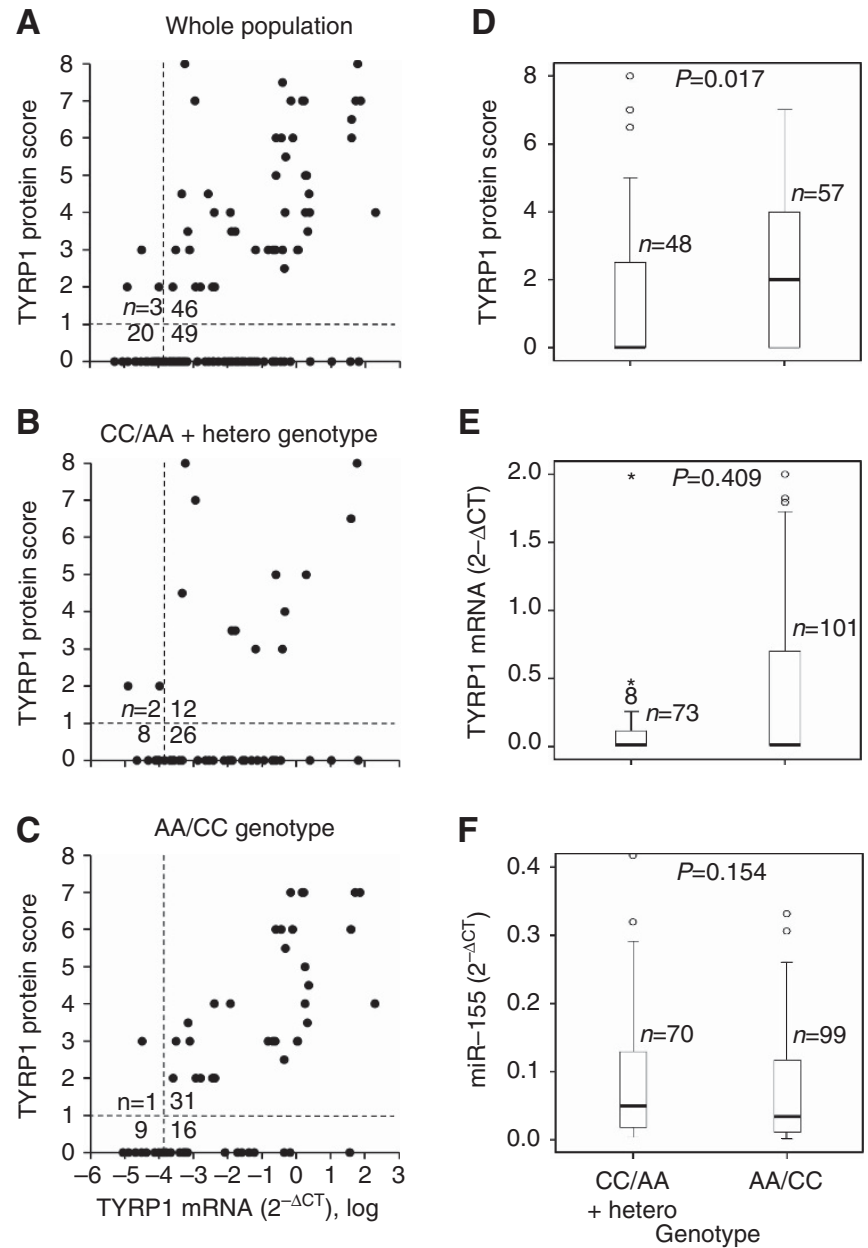

Figure 3. Assessment of TYRP1 mRNA/protein and miR-155 levels in melanoma metastases accounting for SNPs. Correlation between TYRP1 mRNA expression and protein score in melanoma metastases examining (A) the whole population, (B) the 'match' and (C) the 'mismatch' genotype groups. The TYRP1 mRNA level was measured by RT-qPCR and expressed in $\log _{10}$ scale; significant expression was considered to be above the optimal cutoff regarding OS $\left(2^{-\Delta C T}=0.00015\right)$. The TYRP1 protein expression was evaluated by $\mathrm{IHC}$; scores above a value of 2 were considered as positive. The number of tissues $(n)$ with low $\left(2^{-\Delta C T}<0.00015\right)$ and high $\left(2^{-\Delta C T}>0.00015\right)$ TYRP1 mRNA and with $($ score $=0)$ and without (score $=2-8$ ) immunostaining is indicated in each quadrant separately. Expression levels of (D) TYRP1 protein, (E) TYRP1 mRNA and (F) miR155 in the 'match' (CC/AA + hetero) vs the 'mismatch' (AA/CC) genotype groups. Data are presented as box plot: thick line is the median, bottom and top of the box are, respectively, the first and the third quartiles, whiskers indicate the minimum and maximum values, o are outliers and * shows the extreme values. Significance was assessed by Mann-Whitney test. 
supporting that miR-155 may induce mRNA decay and even more repress translation as $68 \%$ (26 out of 38 ) of tissues with positive TYRP1 mRNA were negative for protein (Figure 3B). In contrast, in the 'mismatch' group, miR-155 and TYRP1 mRNA were not correlated $(P=0.853)$, whereas TYRP1 mRNA and protein were $(P<0.001$; Figure $4 \mathrm{~B})$, validating the weak impact of miR-155 in this case, where the discrepancy between mRNA and protein dropped to only $34 \%$ (16 out of 47 ) of samples (Figure $3 \mathrm{C}$ ). Of note, comparing TYRP1 mRNA and MITF mRNA levels, we confirmed significant correlations in both groups.

Prognostic values of TYRP1 protein and mRNA according to SNPs. Finally, we examined the prognostic value of TYRP1 protein and mRNA by determining the correlation between positive (score $=2-8$ ) vs negative $($ score $=0$ ) protein expression (IHC), high $\left(2^{-\Delta \mathrm{CT}}>0.00015\right) v s$ low $\left(2^{-\Delta \mathrm{CT}}<0.00015\right)$ mRNA expression (RT-qPCR) and patient survival. Optimal cutoff for TYRP1 mRNA has been calculated at 20th percentile by using Cutoff Finder software. Unlike TYRP1 mRNA, TYRP1 protein did not correlate with OS using the whole population (Figure 5A and B). On the other hand, the significant correlation between TYRP1 mRNA and survival was lost when the 'match' group (homo CC/AA plus hetero genotype population) was considered (Figure 5C). The TYRP1 protein did not correlate with OS in this group (Figure 5D). In contrast, both protein and mRNA highly correlated with survival in the 'mismatch' group (homo AA/CC genotype population) (Figure 5E and F).

\section{DISCUSSION}

The lack of correlation between TYRP1 mRNA and protein expression and their differential relation with melanoma prognosis that we observed and reported in melanoma metastases (Journe et al, 2011; El Hajj et al, 2013) prompted us to further examine the mechanisms underlying the regulation of TYRP1 protein expression.

The TYRP1 is an enzyme involved in pigmentation whose expression is stimulated by the microphthalmia-associated transcription factor (MITF), the master regulator of cell proliferation,

A

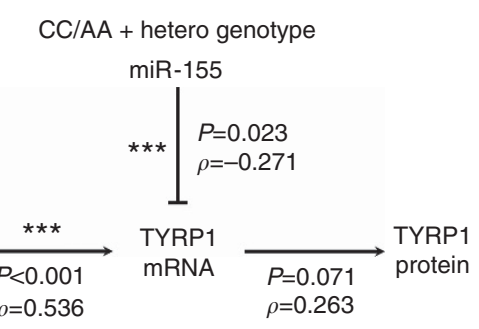

B

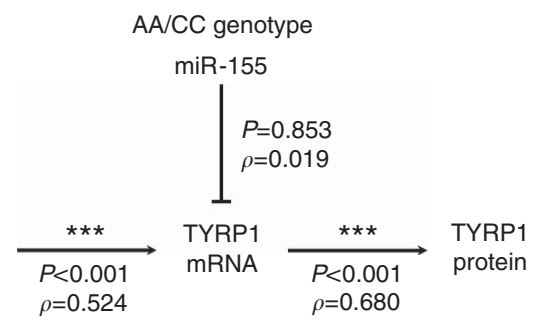

Figure 4. Schematic representation of differential TYRP1 regulation in melanoma metastases accounting for SNPs. The SNPs are proposed to induce miR-155 regulation of TYRP1 in (A) 'match' genotype (CC/

$A A+$ hetero) or disrupt it in (B) 'mismatch' genotype (AA/CC), whereas they do not affect correlation between TYRP1 mRNA and MITF mRNA. Significant correlation between variables was assessed using Spearman's rho test. ${ }^{\star \star \star} P<0.001$. differentiation and melanogenesis (Carreira et al, 2005). As we found a significant correlation between TYRP1 and MITF mRNA expression, the cause of the discrepancy between TYRP1 mRNA and protein is likely to occur at a post-transcriptional level.

A key aspect of the regulation of gene expression is the control of mRNA translation and degradation. Destabilisation of target mRNA by miRNAs is the predominant reason for the reduction of protein synthesis (Ruike et al, 2008). However, miRNAs can also directly repress translation of hundreds of genes without noticeable changes in their corresponding mRNA levels (Selbach et al, 2008; Gu and Kay, 2010).

By performing in silico analysis with microRNA.org (http:// www.microrna.org/) we found that $3^{\prime}$ UTR of TYRP1 mRNA contains, among others, three putative miR-155 binding sites (data not shown). Interestingly, Li et al (2012) discovered that two of these sites show SNP common variants rs683 and rs910 that may induce or disrupt miR-155 regulation and that are differentially distributed among human population following their latitude of residence. Moreover, the efficiency of miRNA-mRNA pairing can be influenced by the presence of SNPs within the miRNA binding sites of mRNA $3^{\prime}$ UTR (Jin and Lee, 2013), thus altering transcript and protein abundance (Gamazon et al, 2012). Hence, based on these data, our study aimed to determine whether miR-155 could specifically regulate TYRP1 translation when accounting for these SNPs.
A

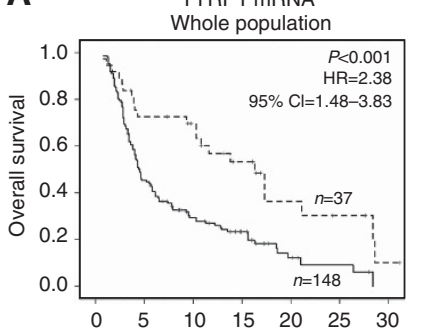

C

E

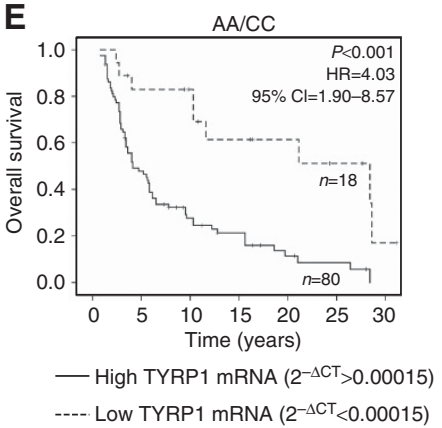

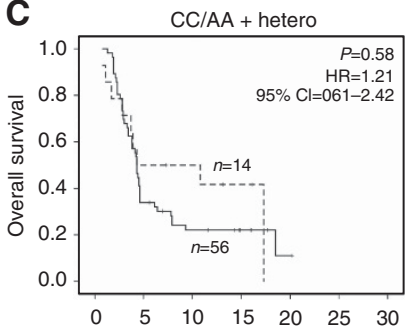

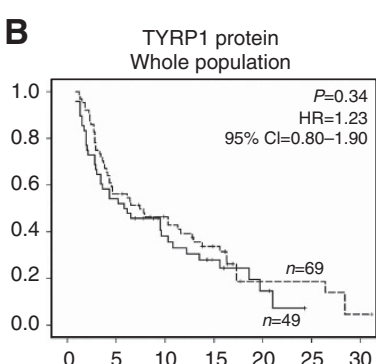

D $\mathrm{CC} / \mathrm{AA}+$ hetero

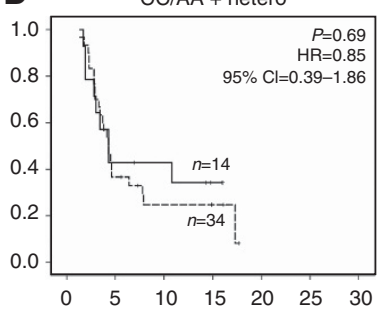

$\mathbf{F}$

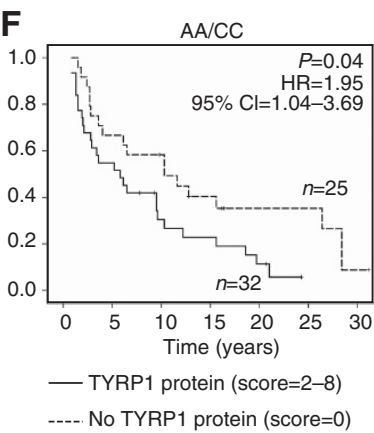

Figure 5. Correlation of TYRP1 mRNA/protein with patients' survival according to SNPs. Survival curves for TYRP1 mRNA and protein in the whole population (A and $\mathbf{B}$ ) and in 'match' (C and $\mathbf{D}$ ) and 'mismatch' ( $E$ and $\mathbf{F}$ ) groups. The OS curves (Kaplan-Meier) were determined according to high/low levels for mRNA with $2^{-\Delta C T}=0.00015$ as cutoff (RT-qPCR, left panels) and positive/negative immunostaining for protein (IHC, right panels). Cox regression was used to calculate $P$-values, hazard ratio $(\mathrm{HR})$ and $95 \%$ confidence interval $(\mathrm{Cl})$.

The symbol ' + ' indicates patients alive at the time of analysis. 
We compared expression levels of TYRP1 mRNA (RT-qPCR) and protein (western blotting) in two melanoma cell lines with different genotypes at rs683/rs910 (MM031 having AA/CC and ME1402 carrying CC/AA), transfected with increasing concentrations of pre-miR-155. Under these conditions and in the MM031 cell line, we found that the expression of both TYRP1 mRNA and protein decayed similarly with a rather low slope, whereas in ME1402 cells, the decrease of TYRP1 mRNA followed a higher slope that was much steeper for the protein, suggesting that, in the latter line, miR-155 has a stronger ability to bind and degrade mRNA and, consequently, inhibit translation from residual mRNA. This is in accordance with other studies reporting that a miRNA-mRNA perfect seed pairing degraded mRNA and blocked its translation into protein, whereas target mRNA sites with imperfect base pairing were weakly regulated by miRNAs (Valencia-Sanchez et al, 2006).

We then conducted a validation study in 192 skin and lymph node melanoma metastases assessing TYRP1 mRNA, TYRP1 protein and miR-155 expression as well as genotype at the $3^{\prime} \mathrm{UTR}$ of TYRP1 mRNA, and examined various correlations accounting for SNPs. We first checked for a possible correlation between miR155 and patient survival in these samples. Although miR-155 may function as an oncogene in melanoma because its expression level was associated with Breslow thickness (Grignol et al, 2011), which is known to highly correlate with shorter survival, it may also act as a tumour suppressor (Levati et al, 2009) and was associated with longer survival in a small melanoma cohort (Segura et al, 2010). In our 192 samples, using an optimal cutoff of $2^{-\Delta \mathrm{CT}}=0.0522$ (median) for miR-155 as assessed with Cutoff Finder software, we found that miR-155 levels did not correlate with OS whatever SNP-defined subpopulation we examined. Even in the 'match' group where one could have expected a correlation with OS as miR-155 inversely correlated with TYRP1 mRNA $(P=0.023$, Spearman's rho test), we did not find any significant correlation $(P=0.090$, Cox regression).

Polymorphisms have also been reported to be associated with cancers and proposed as cancer biomarkers (Preskill and Weidhaas, 2013). For example, Brendle et al (2008) identified a SNP within the $3^{\prime} \mathrm{UTR}$ of ITGB4 mRNA associated with poorer breast cancer survival. Accordingly, we could not observe such a correlation using the SNPs at TYRP1 mRNA $3^{\prime}$ UTR described above $(P=0.283$, Cox regression). In contrast, we could clearly identify two polymorphic groups ('match' and 'mismatch') having different expression levels of TYRP1 protein, but similar TYRP1 mRNA or miR-155, supporting the fact that TYRP1 protein was differentially affected by miR-155 in a SNP-dependent manner.

In accordance with $\mathrm{Li}$ et al (2012) reporting that the heterozygous genotype has one $\mathrm{C} / \mathrm{A}$ allele that may allow miR155 regulation, and with our observation that TYRP1 protein expression did not significantly differ between the heterozygous and the homozygous CC/AA groups, we combined both genotypes to form the 'match' group, AA/CC homozygous genotype being the 'mismatch' one. The latter group had higher TYRP1 protein expression that significantly correlated with TYRP1 mRNA, supporting that the low binding capacity of miR-155 to its target sites did not prevent translation, whereas in the 'match' group, TYRP1 protein levels were comparatively lower and not correlated with mRNA levels, sustaining that, in this case, the high binding capacity of miR-155 to TYRP1 mRNA can hamper protein synthesis. Thus, in the 'match' group, because miR-155 can more efficiently bind TYRP1 mRNA and interfere with its translation into protein, $67 \%$ of samples exhibited significant TYRP1 mRNA levels but no protein. This may be viewed as an additional mechanism regulating TYRP1 protein expression at the post-transcriptional level and, subsequently, melanogenesis.

To further support our findings, we examined TYRP1 protein expression by IHC (positive immunostaining validated with three different antibodies recognising distinct epitopes) and found a significant correlation between the presence of TYRP1 protein (score $=2-8)$ in tumour tissues and a shorter OS but only in the 'mismatch' group where TYRP1 mRNA and protein correlated. This may explain our previous observations reporting the lack of correlation between TYRP1 protein and mRNA in skin metastases (Journe et al, 2011), and between TYRP1 protein expression and survival (Bolander et al, 2008; Journe et al, 2011), as well as the absence of TYRP1 protein detection in the vertical/invasive growth phase of primary lesions (Fang et al, 2001).

In conclusion, based on evidence from melanoma cell lines and a validation in skin and lymph node metastases, we demonstrate a differential post-transcriptional regulation of TYRP1 by miR-155 according to the SNPs rs683 and rs910. Such polymorphisms affect TYRP1 mRNA regulation by miR-155 and subsequent translation into protein. This explains the discrepancy between the prognostic value of TYRP1 mRNA and protein expression; the latter only correlates with patient survival when stratified for the $3^{\prime}$ UTR SNPs in the miR-155 binding site of TYRP1 mRNA. Therefore, in the context of efforts currently done to identify high-risk metastatic melanoma patients with a longer life expectancy, the evaluation of TYRP1 may be used to refine the initial prognosis defined at the time of primary or to establish one for those with unknown primaries, and may dictate a different management of the advanced disease.

\section{ACKNOWLEDGEMENTS}

This study received financial support from the 'MEDIC Foundation', 'Les Amis de l'Institut Bordet', the 'Fondation LambeauMarteaux', the 'EORTC-Melanoma Group', 'Wallonie-Bruxelles International', the INCa PAIR Melanoma program, the 'Départements Grand Ouest de Ligue National contre le Cancer' and the 'Société Française de Dermatologie'. Petra El Hajj is the recipient of a fellowship from the Lebanese National Council for Scientific Research and the Lebanese University.

\section{REFERENCES}

Alonzo TA (2005) Standards for reporting prognostic tumor marker studies. J Clin Oncol Off J Am Soc Clin Oncol 23: 9053-9054.

Balch CM, Gershenwald JE, Soong S-J, Thompson JF, Atkins MB, Byrd DR, Buzaid AC, Cochran AJ, Coit DG, Ding S, Eggermont AM, Flaherty KT, Gimotty PA, Kirkwood JM, McMasters KM, Mihm Jr MC, Morton DL, Ross MI, Sober AJ, Sondak VK (2009) Final version of 2009 AJCC melanoma staging and classification. J Clin Oncol Off J Am Soc Clin Oncol 27: 6199-6206.

Bolander A, Agnarsdóttir M, Strömberg S, Ponten F, Hesselius P, Uhlen M, Bergqvist M (2008) The protein expression of TRP-1 and galectin-1 in cutaneous malignant melanomas. Cancer Genomics Proteomics 5: 293-300.

Brendle A, Lei H, Brandt A, Johansson R, Enquist K, Henriksson R, Hemminki K, Lenner P, Försti A (2008) Polymorphisms in predicted microRNA-binding sites in integrin genes and breast cancer: ITGB4 as prognostic marker. Carcinogenesis 29: 1394-1399.

Budczies J, Klauschen F, Sinn BV, Győrffy B, Schmitt WD, Darb-Esfahani S, Denkert C (2012) Cutoff Finder: a comprehensive and straightforward web application enabling rapid biomarker cutoff optimization. PLoS One 7: e51862.

Bustin SA, Benes V, Garson JA, Hellemans J, Huggett J, Kubista M, Mueller R, Nolan T, Pfaffl MW, Shipley GL, Vandesompele J, Wittwer CT (2009) The MIQE guidelines: minimum information for publication of quantitative real-time PCR experiments. Clin Chem 55: 611-622.

Carreira S, Goodall J, Aksan I, La Rocca SA, Galibert M-D, Denat L, Larue L, Goding CR (2005) Mitf cooperates with Rb1 and activates p21Cip1 expression to regulate cell cycle progression. Nature 433: 764-769. 
El Hajj P, Journe F, Wiedig M, Laios I, Salès F, Galibert M-D, Van Kempen LC, Spatz A, Badran B, Larsimont D, Awada A, Ghanem G (2013) Tyrosinase-related protein $1 \mathrm{mRNA}$ expression in lymph node metastases predicts overall survival in high-risk melanoma patients. Br J Cancer 108(8): 1641-1647.

Fang D, Hallman J, Sangha N, Kute TE, Hammarback JA, White WL, Setaluri V (2001) Expression of microtubule-associated protein 2 in benign and malignant melanocytes: implications for differentiation and progression of cutaneous melanoma. Am J Pathol 158: 2107-2115.

Gamazon ER, Ziliak D, Im HK, LaCroix B, Park DS, Cox NJ, Huang RS (2012) Genetic architecture of microRNA expression: implications for the transcriptome and complex traits. Am J Hum Genet 90: 1046-1063.

Ghanem G, Fabrice J (2011) Tyrosinase related protein 1 (TYRP1/gp75) in human cutaneous melanoma. Mol Oncol 5: 150-155.

Gould Rothberg BE, Bracken MB, Rimm DL (2009) Tissue biomarkers for prognosis in cutaneous melanoma: a systematic review and meta-analysis. J Natl Cancer Inst 101: 452-474.

Grignol V, Fairchild ET, Zimmerer JM, Lesinski GB, Walker MJ, Magro CM, Kacher JE, Karpa VI, Clark J, Nuovo G, Lehman A, Volinia S, Agnese DM, Croce CM, Carson 3rd WE (2011) miR-21 and miR-155 are associated with mitotic activity and lesion depth of borderline melanocytic lesions. $\mathrm{Br}$ J Cancer 105: 1023-1029.

Gu S, Kay MA (2010) How do miRNAs mediate translational repression? Silence 1: 11.

Jin Y, Lee CGL (2013) Single nucleotide polymorphisms associated with microRNA Regulation. Biomolecules 3: 287-302.

Journe F, Id Boufker H, Van Kempen L, Galibert M-D, Wiedig M, Salès F, Theunis A, Nonclercq D, Frau A, Laurent G, Awada A, Ghanem G (2011) TYRP1 mRNA expression in melanoma metastases correlates with clinical outcome. Br J Cancer 105: 1726-1732.

Kashani-Sabet M, Venna S, Nosrati M, Rangel J, Sucker A, Egberts F, Baehner FL, Simko J, Leong SPL, Haqq C, Hauschild A, Schadendorf D, Miller 3rd JR, Sagebiel RW (2009) A multimarker prognostic assay for primary cutaneous melanoma. Clin Cancer Res Off J Am Assoc Cancer Res 15: 6987-6992.

Levati L, Alvino E, Pagani E, Arcelli D, Caporaso P, Bondanza S, Di Leva G, Ferracin M, Volinia S, Bonmassar E, Croce CM, D'Atri S (2009) Altered expression of selected microRNAs in melanoma: antiproliferative and proapoptotic activity of miRNA-155. Int J Oncol 35: 393-400.

Li J, Liu Y, Xin X, Kim TS, Cabeza EA, Ren J, Nielsen R, Wrana JL, Zhang Z (2012) Evidence for positive selection on a number of microRNA regulatory interactions during recent human evolution. PLoS Genet 8: e1002578.
McShane LM, Altman DG, Sauerbrei W, Taube SE, Gion M, Clark GM (2005) REporting recommendations for tumour MARKer prognostic studies (REMARK). Br J Cancer 93: 387-391.

Meyer S, Fuchs TJ, Bosserhoff AK, Hofstadter F, Pauer A, Roth V, Buhmann JM, Moll I, Anagnostou N, Brandner JM, Ikenberg K, Moch H, Landthaler M, Vogt T, Wild PJ (2012) A seven-marker signature and clinical outcome in malignant melanoma: a large-scale tissue-microarray study with two independent patient cohorts. PLoS One 7(6): e38222.

Osman A (2012) MicroRNAs in health and disease-basic science and clinical applications. Clin Lab 58: 393-402.

Primot A, Mogha A, Corre S, Roberts K, Debbache J, Adamski H, Dreno B, Khammari A, Lesimple T, Mereau A, Goding CR, Galibert MD (2010) ERK-regulated differential expression of the Mitf $6 \mathrm{a} / \mathrm{b}$ splicing isoforms in melanoma. Pigment Cell Melanoma Res 23: 93-102.

Preskill C, Weidhaas JB (2013) SNPs in microRNA binding sites as prognostic and predictive cancer biomarkers. Crit Rev Oncog 18: 327-340.

Reich DE, Gabriel SB, Altshuler D (2003) Quality and completeness of SNP databases. Nat Genet 33: 457-458.

Ruike Y, Ichimura A, Tsuchiya S, Shimizu K, Kunimoto R, Okuno Y, Tsujimoto G (2008) Global correlation analysis for micro-RNA and mRNA expression profiles in human cell lines. J Hum Genet 53: 515-523.

Sayed D, Abdellatif M (2011) MicroRNAs in development and disease. Physiol Rev 91: 827-887.

Segura MF, Belitskaya-Lévy I, Rose AE, Zakrzewski J, Gaziel A, Hanniford D, Darvishian F, Berman RS, Shapiro RL, Pavlick AC, Osman I, Hernando E (2010) Melanoma microRNA signature predicts post-recurrence survival. Clin Cancer Res Off J Am Assoc Cancer Res 16: 1577-1586.

Selbach M, Schwanhäusser B, Thierfelder N, Fang Z, Khanin R, Rajewsky N (2008) Widespread changes in protein synthesis induced by microRNAs. Nature 455: 58-63.

Valencia-Sanchez MA, Liu J, Hannon GJ, Parker R (2006) Control of translation and mRNA degradation by miRNAs and siRNAs. Genes Dev 20: $515-524$.

This work is published under the standard license to publish agreement. After 12 months the work will become freely available and the license terms will switch to a Creative Commons AttributionNonCommercial-Share Alike 4.0 Unported License 